\begin{tabular}{l} 
O P E R A T I O N S R E S E A R C H A N D D E C I S I O N S \\
\hline No. 3
\end{tabular}

DOI: $10.37190 /$ ord200306

\title{
INVENTORY MODEL FOR DETERIORATING ITEMS WITH NEGATIVE EXPONENTIAL DEMAND, PROBABILISTIC DETERIORATION, AND FUZZY LEAD TIME UNDER PARTIAL BACK LOGGING
}

\author{
NABENDU SEN*, SUMIT SAHA \\ Department of Mathematics, Assam University, Silchar, 788 011, Assam, India
}

\begin{abstract}
The effect of lead time plays an important role in inventory management. It is also important to study the optimal strategies when the lead time is not precisely known to the decision-makers. This paper aimed to examine the inventory model for deteriorating items with fuzzy lead time, negative exponential demand, and partially backlogged shortages. This model is unique in its nature due to probabilistic deterioration along with fuzzy lead time. The fuzzy lead time was assumed to be triangular, parabolic, trapezoidal numbers. The graded mean integration representation method was used for the defuzzification purpose. Three different types of probability distributions, namely uniform, triangular and beta were used for rate of deterioration to find optimal time and associated total inventory cost. The developed model was validated numerically, and values of optimal time and total inventory cost are given in a tabular form, corresponding to different probability distributions and fuzzy lead-time. The sensitivity analysis was performed on the variation of key parameters to observe its effect on the developed model.
\end{abstract}

Keywords: fuzzy lead time, shortages, partially backlogged, exponentially declining demand, probabilistic deterioration

\section{Introduction}

Conventional inventory models are formulated under the assumption that the time gap between placing an order and receiving goods is negligible. This indicates that suppliers of goods are quite near retailers and always ready to make the required quantity of goods available to its customers in fraction of time. But, in practice, the situation is not so fast and simple with no waste of time. The reality lies with the fact that there

*Corresponding author, email address: nsen08@yahoo.com Received 17 May 2019, accepted 23 August 2020 
always remains a time gap between placing an order and receiving items which are produced at different production units spread over various places. Moreover, this time gap is not exactly known since some commodities whose demand is gradually decreasing but those commodities have a section of customers. Hence, non-zero lead-time in particular fuzzy lead time plays a vital role in inventory modelling. As a result, this type of problem in inventory management draws attraction of researchers and practitioners throughout the world. Over the years, the inventory problem for deteriorating item has undergone substantial improvement due to the addition of new assumptions on the nature of deterioration and other parameters. Among those, to get recent development in research in inventory problems, one may refer to the works of $[19,20,31,2]$.

\section{Survey of literature}

In this section, some of the major works done in the field of inventory management in respect of various parameters in the last few years, both in crisp and fuzzy environments, are discussed. Modelling any inventory problem in the absence of non-zero lead time and deterioration cannot reflect the actual scenarios. It is well known that any item, when purchased/produced, never remains the same for a long time. With time, the item changes due to spoilage, deterioration, weather condition, and other factors. So, naturally, the question arises how to maintain the stock of items for its further use, and to optimize total cost. Taking this fact into consideration, researchers developed inventory models for optimal solutions. To deal with the issue of probabilistic deterioration, a model was developed in [17]. During the formulation of the inventory model in [1], three parameters of the Weibull-distributed deterioration and selling price dependent demand are incorporated. Giving importance on time-price dependent demand [23], formulate a problem for deteriorating items in which lead time is considered as zero. Same authors [24] discuss an inventory model with price-sensitive demand for deteriorating items under full advance payment for availing discount and solve it numerically. Recently, Khurana et al. [8] intended to minimize inventory cost for deteriorating items when the demand for such type of items is variable with allowable shortages. In the literature, there is the consideration of demand as a ramp type function of time and twoparameter Weibull-distributed deterioration in formulating inventory model [6]. One can easily see that the model was developed for deteriorating items with time-dependent demand under inflation when supplier credit is linked with order quantity [30]. Also, the effect of inflation in the optimal solution of an inventory system for permissible items is studied where demand is taken as a quadratic function of time and constant rate of deterioration [18]. Considering fluctuating demand as a function of time, an inventory model for deteriorating items was developed under permissible delay in payment [22]. Apart from the aforesaid models, there are good numbers of research papers where the 
effect of lead time is ignored despite having a significant role in inventory decision-making policies. The consideration of other major factors, like demand, deterioration, and display cost, permissible delay in payment, etc., are frequently available in current literature. The contribution in inventory management under fuzzy environment is worth mentioning in [4] and in [28]. There are numerous papers where inventory models are developed in the fuzzy environment under various assumptions. The consideration of fuzzy parameters in mathematical modelling of inventory system is available in [15, 13, 3, 9]. Following Kazemi et al. [10], a table is made to show a comparison of contributions by different authors.

Table 1. Classification of the literature

\begin{tabular}{|c|l|l|l|l|}
\hline Reference & Environment & \multicolumn{1}{|c|}{ Demand } & \multicolumn{1}{|c|}{ Deterioration } & $\begin{array}{c}\text { Lead } \\
\text { time }\end{array}$ \\
\hline$[5]$ & crisp & stockdependent (non-exponential) & constant & zero \\
\hline$[7]$ & fuzzy & constant (non-exponential) & constant & constant \\
\hline$[11]$ & crisp & time dependent (non-exponential) & time dependent & zero \\
\hline$[12]$ & crisp & inflation induced (non-exponential) & Weibull distribution & zero \\
\hline$[14]$ & fuzzy & $\begin{array}{l}\text { display inventory dependent } \\
\text { (non-exponential) }\end{array}$ & no & zero \\
\hline$[16]$ & crisp & price sensitive (non-exponential) & constant & zero \\
\hline$[21]$ & fuzzy & constant (non-exponential) & constant & zero \\
\hline$[25]$ & crisp & time dependent (exponential) & time dependent & zero \\
\hline$[26]$ & fuzzy & price dependent (non-exponential) & constant & zero \\
\hline$[27]$ & fuzzy & constant & fuzzy number & zero \\
\hline$[29]$ & crisp & inventory dependent (non-exponential) & time dependent & zero \\
\hline Summary & $\begin{array}{l}6 \text { crisp } \\
5 \text { fuzzy }\end{array}$ & $\begin{array}{l}10 \text { non-exponential } \\
1 \text { exponential }\end{array}$ & $\begin{array}{l}1 \text { probabilistic } \\
10 \text { non-probabilistic }\end{array}$ & $\begin{array}{l}1 \text { non zero } \\
10 \text { zero }\end{array}$ \\
\hline Present study & & negative exponential & probabilistic & fuzzy \\
\hline
\end{tabular}

To the best knowledge of the authors, and as shown in the above contributions table, there is no paper where the discussion of inventory model with probabilistic deterioration and fuzzy lead-time simultaneously took place. The present study is motivated by the work conducted in [28]. The main purpose of this study is to develop a mathematical model with a set of assumptions emphasizing probabilistic deterioration and fuzzy lead time, and simultaneously which the problems existing in the real world.

\section{Notations and assumptions}

\section{Assumptions}

- planning horizon is infinite,

- demand rate is exponentially decreasing, 
- shortages are allowed and partially backlogged,

- holding cost is independent of time,

- deterioration is probabilistic in nature,

- there is no repair of deteriorating items,

- lead time is non-zero and fuzzy in nature.

\section{Notations}

$h=a \quad-$ holding cost per unit time, $a>0$, \$/unit

$\theta(t)=\theta t \quad-$ deterioration rate $0<\theta<1$

$D(t)=A \mathrm{e}^{-\alpha t}-$ demand function

$c_{0} \quad-$ ordering cost, $\$$

$c_{\mathrm{s}} \quad-$ shortage cost, \$/unit

c $\quad-$ unit cost of the item, \$/unit

$L \quad$ - lead time, months

$\tilde{L} \quad-$ fuzzy lead time, months

$T \quad-$ cycle length, month

$I_{0} \quad-$ maximum inventory level in $(0, T)$

$S \quad-$ lost sale cost per unit, \$/unit

$c_{d} \quad-$ unit deterioration cost, \$/unit

$t_{1} \quad-$ decision variable, time of replenishment, months

TC objective, total cost of the inventory system, \$

\section{Mathematical formulation of the models}

For the crisp environment, it is assumed that the replenishment rate is finite and time gap of placing and receiving order is not fixed uncertain and that deterioration takes place randomly. So, as based on the above-mentioned assumptions, the differential equations representing the proposed inventory system as

$$
\frac{d I_{1}(t)}{d t}+\theta t I_{1}(t)=-A \mathrm{e}^{-\alpha t} \text { with } I_{1}(t)=I_{0}
$$

The rate of change of the inventory during the shortage period $\left[t_{1}, t_{2}\right]$ is governed by the differential equation

$$
\frac{d I_{2}(t)}{d t}=-A \beta \mathrm{e}^{-\alpha t}, \quad t_{1} \leq t \leq t_{2}, \quad I_{2}\left(t_{1}\right)=0
$$


The inventory level changes in $\left[t_{2}, T\right]$ is due to demand and deterioration

$$
\frac{d I_{3}(t)}{d t}+\theta t I_{3}(t)=-A \mathrm{e}^{-\alpha t} \quad \text { with } \quad I_{3}\left(t_{2}\right)=I_{0}
$$

The solution of (1) gives

$$
I_{1}(t)=k_{1} \mathrm{e}^{-\theta t^{2} / 2}+\frac{A \mathrm{e}^{-\alpha t}}{\theta t-\alpha}
$$

Applying $I_{1}(0)=I_{0}$, the above expression gives

$$
k_{1}=I_{0}+\frac{A}{\alpha} S_{0} I_{1}(t)=\left(I_{0}+\frac{A}{\alpha}\right) \mathrm{e}^{-\theta t^{2} / 2}+\frac{A \mathrm{e}^{-\alpha t}}{\theta t-\alpha}
$$

The solution of equation (2) gives

$$
I_{2}(t)=-A \beta\left(-\frac{\mathrm{e}^{-\alpha t}}{\alpha}\right)+k_{2}
$$

On applying $I_{2}\left(t_{1}\right)=0$, we get $k_{2}=-\frac{A \beta}{\alpha} \mathrm{e}^{-\alpha t_{1}}$ implying

$$
I_{2}(t)=\frac{A \beta}{\alpha}\left(\mathrm{e}^{-\alpha t}-\mathrm{e}^{-\alpha t_{1}}\right)
$$

The solution of equation (3) gives

$$
I_{3}(t)=k_{3} \mathrm{e}^{-\theta t^{2} / 2}+\frac{A \mathrm{e}^{-\alpha t}}{\theta t-\alpha}
$$

At $t=t_{2}, I_{3}(t)=I_{0}$, giving

$$
k_{3}=\mathrm{e}^{\theta t_{2}^{2} / 2}\left(I_{0}-\frac{A \mathrm{e}^{-\alpha t_{2}}}{\theta t_{2}-\alpha}\right)
$$

using $k_{3}$ in equation (7), 


$$
I_{3}(t)=\left(I_{0}-\frac{A e^{-\alpha t_{2}}}{\theta t_{2}-\alpha}\right) \mathrm{e}^{\theta\left(t_{2}^{2}-t^{2}\right)}+\frac{A \mathrm{e}^{-\alpha t}}{\theta t-\alpha}
$$

But $t_{2}-t_{1}=L=>t_{2}=t_{1}+L$. So, equation (9) becomes

$$
I_{3}(t)=\left(I_{0}-\frac{A \mathrm{e}^{t_{1}+L}}{\theta\left(t_{1}+L\right)-\alpha}\right) \mathrm{e}^{\theta\left(\left(t_{1}+L\right)^{2}-t^{2}\right) / 2}+\frac{A \mathrm{e}^{-\alpha t}}{\theta t-\alpha}
$$

The remaining quantities are

- backlogged quantity

$$
B Q=\int_{t_{1}}^{t_{2}} I_{2}(t) d t=\frac{A \beta \mathrm{e}^{-\alpha t_{1}}}{\alpha^{2}}\left(\mathrm{e}^{-\alpha L}-1+L \alpha\right)
$$

- lost sale

$$
\int_{t_{1}}^{t_{2}} D(t) d t-\int_{t_{1}}^{t_{2}} I_{2}(t) d t=\frac{A \mathrm{e}^{-\alpha t_{1}}}{\alpha^{2}}\left((\beta-\alpha)\left(\mathrm{e}^{-\alpha L}-1\right)+\beta \alpha L\right)
$$

- deteriorated quantity

$$
Q_{d}=2 I_{0}+\frac{A}{\alpha}\left(\mathrm{e}^{-\alpha T}-1\right)+\frac{A}{\alpha} \mathrm{e}^{-\alpha t_{1}}\left(1-\mathrm{e}^{-\alpha L}\right)
$$

- holding cost $H C=H C$ in $\left[0, t_{1}\right]+H C$ in $\left[t_{1}, T\right]$

$$
\begin{aligned}
H C= & h \int_{0}^{t_{1}} I_{1}(t) d t+h \int_{t_{1}}^{T} I_{3}(t) d t=h\left(t_{1}-\frac{A}{\alpha}\left(\frac{\theta}{\alpha}-\alpha\right) \frac{t_{1}^{2}}{2}-\frac{1}{6}\left(I_{0}-\frac{A \theta}{\alpha}\right) t_{1}^{3}\right) \\
& +h\left(\left(X_{1}-\frac{A}{\alpha}\right)\left(T-t_{1}-L\right)+\frac{A}{2}\left(\frac{\theta}{\alpha^{2}}+1\right)\left(T^{2}-t_{1}^{2}-L^{2}-2 t_{1} L\right)\right. \\
& \left.-\frac{\theta}{3}\left(\frac{X_{1}}{2}+\frac{A}{\alpha}\right)\left(T^{3}-t_{1}^{3}-3 t_{1}^{2} L-3 t_{1} L^{2}-T^{3}\right)\right)
\end{aligned}
$$

where 


$$
X_{1}=\left(I_{0}-\frac{A \mathrm{e}^{t_{1}+L}}{\theta\left(t_{1}+L\right)-\alpha}\right) \mathrm{e}^{-\theta\left(t_{1}+L\right)^{2}}
$$

- deteriorated cost

$$
D C=C_{d} Q_{d}=C_{d}\left(2 I_{0}+\frac{A}{\alpha}\left(\mathrm{e}^{-\alpha T}-1\right)+\frac{A}{\alpha} \mathrm{e}^{-\alpha t_{1}}\left(1-\mathrm{e}^{\alpha L}\right)\right)
$$

- total shortages during $\left[t_{1}, t_{2}\right]$

$$
\int_{t_{1}}^{t_{2}} D(t) d t=-\frac{A}{\alpha} \mathrm{e}^{-\alpha t_{1}}\left(\mathrm{e}^{-\alpha L}-1\right)
$$

- shortage cost

$$
S C=-c_{s} \frac{A}{\alpha} \mathrm{e}^{-\alpha t_{1}}\left(\mathrm{e}^{-\alpha L}-1\right)
$$

- purchase cost

$$
P C=c\left(2 I_{0}-B Q\right)=c\left(2 I_{0}-\frac{A \beta}{\alpha^{2}} \mathrm{e}^{-\alpha t_{1}}\left(\mathrm{e}^{-\alpha L}+L \alpha-1\right)\right)
$$

- lost cost sale

$$
L S C=S \frac{A \mathrm{e}^{-\alpha t_{1}}}{\alpha^{2}}\left(\left(e^{-\alpha L}-1\right)(\beta-\alpha)+\alpha \beta L\right)
$$

Total cost of the inventory system is as follows

$$
\begin{gathered}
T C=O C+P C+H C+S C+L S C+D C \\
T C=k_{0}+k_{1} t_{1}+k_{2} t_{1}^{2}+k_{3} t_{1}^{3}+k_{4} t_{1}^{4}+M_{1} L+M_{2} L^{2}+M_{3} L^{3}+M_{4} L^{4} \\
+N_{1} L t_{1}+N_{2} L t_{1}^{2}+N_{3} L t_{1}^{3}+N_{4} L^{2} t_{1}+N_{5} L^{2} t_{1}^{2}+N_{6} L^{3} t_{1}-A c_{d} T
\end{gathered}
$$

where 


$$
\begin{gathered}
k_{0}=2 A+2 C I_{0}+h I_{0}+\frac{h A}{\alpha}+2 c_{d} I_{0} \\
k_{1}=h\left(1+\frac{A}{\alpha}\left(\frac{\theta}{\alpha}-\alpha\right)\right), \quad k_{2}=h\left(\frac{-h}{2 \alpha}\left(\frac{\theta}{\alpha}-\alpha\right)+\frac{I_{0} \theta}{2}+\frac{A}{\alpha}-\frac{A \theta}{\alpha}\right) \\
k_{3}=h\left(\frac{-h}{6}\left(I_{0}-\frac{A \theta}{\alpha}\right)+\frac{A \theta}{2 \alpha}\left(\frac{\theta}{\alpha}-\alpha\right)\right), \quad k_{4}=-h \frac{A \theta^{2}}{2 \alpha} \\
M_{1}=A\left(\frac{h}{\alpha}\left(\frac{\theta}{\alpha}-\alpha\right)-c_{s}+S+c_{d}\right), \quad M_{2}=h\left(\frac{I_{0} \theta}{2}+\frac{A}{\alpha}-\frac{A \theta}{\alpha}\right) \\
N_{1}=2 h\left(\frac{I_{0} \theta}{2}+\frac{A}{\alpha}-\frac{A \theta}{\alpha}\right), \quad N_{2}=\frac{3 h A \theta}{2 \alpha}, \quad N_{3}=-\frac{2 h A \theta^{2}}{\alpha} \\
N_{4}=\frac{3 h A}{\alpha}\left(\frac{\theta}{\alpha}-\alpha\right), \quad N_{5}=-\frac{3 h A \theta^{2}}{\alpha}, \quad N_{6}=-\frac{2 h A \theta^{2}}{\alpha} \\
M_{3} \\
\alpha A_{4}=-\frac{h A \theta^{2}}{2 \alpha} \\
\alpha
\end{gathered}
$$

\section{Numerical results of the model in various environments}

The numerical values of different parameters are given below in appropriate units: $h=2$, $A=100, \beta=0.3, \alpha=0.001, I_{0}=1000, c_{0}=50, c=50, c_{d}=0.75, L=0.34, \theta=0.12$.

Table 2. Values of $\theta$ for various distributions

\begin{tabular}{|c|c|c|c|}
\hline \multirow{2}{*}{$\theta$} & Triangular & Uniform & Beta \\
\cline { 2 - 4 } & $(0.002,0.007,0.3)$ & $(0.002,0.2)$ & $(0.002,0.02)$ \\
\hline
\end{tabular}

Table 3. Values of lead time for various fuzzy numbers

\begin{tabular}{|c|c|c|c|}
\hline \multirow{2}{*}{$\tilde{L}$} & Triangular & Parabolic & Trapezoidal \\
\cline { 2 - 4 } & $(0.33,0.40,0.47)$ & $(0.20,0.3,0.47)$ & $(0.20,0.27,0.33,0.47)$ \\
\hline
\end{tabular}

Due to the complex nature of the cost function, the optimality is checked graphically in crisp and fuzzy environment using MATLAB. The optimal results are listed for three 
different cases for rate of deterioration when it follows triangular, uniform, and beta distribution.

\subsection{Numerical results}

Numerical results in the crisp environment are as follows:

Table 4. Optimal values of $t_{1}$ and $T C$ corresponding to various probability distributions

\begin{tabular}{|c|c|c|c|c|c|}
\hline \multicolumn{2}{|c|}{$\theta$ follows triangular distribution } & \multicolumn{2}{|c|}{$\theta$ follows uniform distribution } & \multicolumn{2}{c|}{$\theta$ follows beta distribution } \\
\hline $\begin{array}{c}t_{1} \\
{[\text { months }]}\end{array}$ & $\begin{array}{c}T C \\
{[\$]}\end{array}$ & $\begin{array}{c}t_{1} \\
{[\text { months }]}\end{array}$ & $\begin{array}{c}T C \\
{[\$]}\end{array}$ & $\begin{array}{c}t_{1} \\
{[\text { months }]}\end{array}$ & $\begin{array}{c}T C \\
{[\$]}\end{array}$ \\
\hline 5 & 7468000 & 5 & 6541000 & 6 & 460000 \\
\hline
\end{tabular}

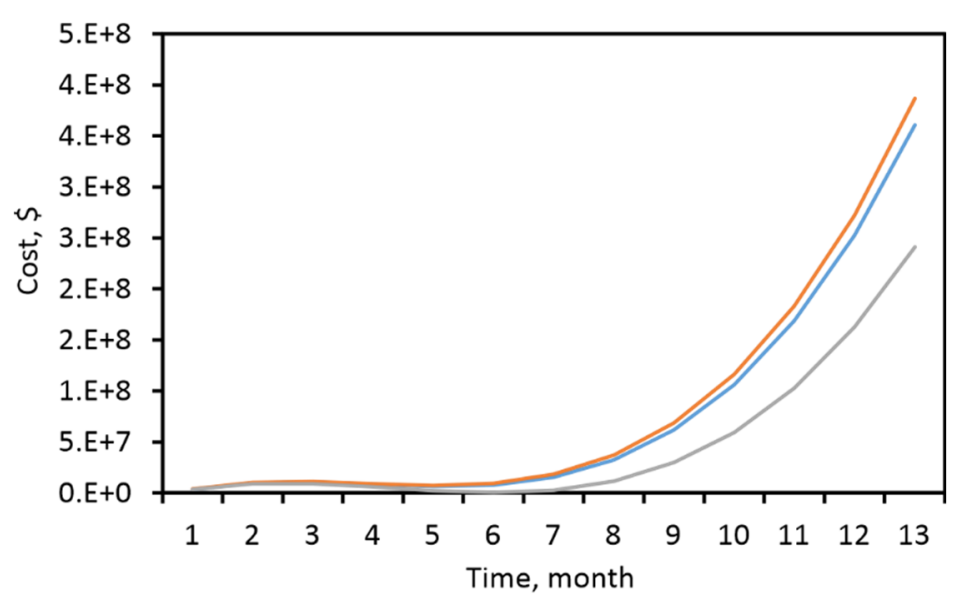

Uniform distribution Triangular distribution

Beta distribution

Fig. 1. Graphical representation of the results presented in Table 4

Numerical result in fuzzy environment are following when the rate of deterioration follows various distributions and lead time is fuzzy numbers:

Table 5. Optimal values corresponding to various fuzzy lead times when the deterioration follows beta distribution

\begin{tabular}{|c|c|c|c|c|c|}
\hline \multicolumn{2}{|c|}{ Triangular fuzzy number } & \multicolumn{2}{|c|}{ Parabolic fuzzy number } & \multicolumn{2}{c|}{ Trapezoidal number } \\
\hline $\begin{array}{c}t_{1} \\
{[\text { months }]}\end{array}$ & $\begin{array}{c}T C \\
{[\$]}\end{array}$ & $\begin{array}{c}t_{1} \\
{[\text { months }]}\end{array}$ & $\begin{array}{c}T C \\
{[\$]}\end{array}$ & $\begin{array}{c}t_{1} \\
{[\text { months }]}\end{array}$ & $\begin{array}{c}T C \\
{[\$]}\end{array}$ \\
\hline 6 & 3194000 & 6 & 129200000 & 6 & -2300000 \\
\hline
\end{tabular}




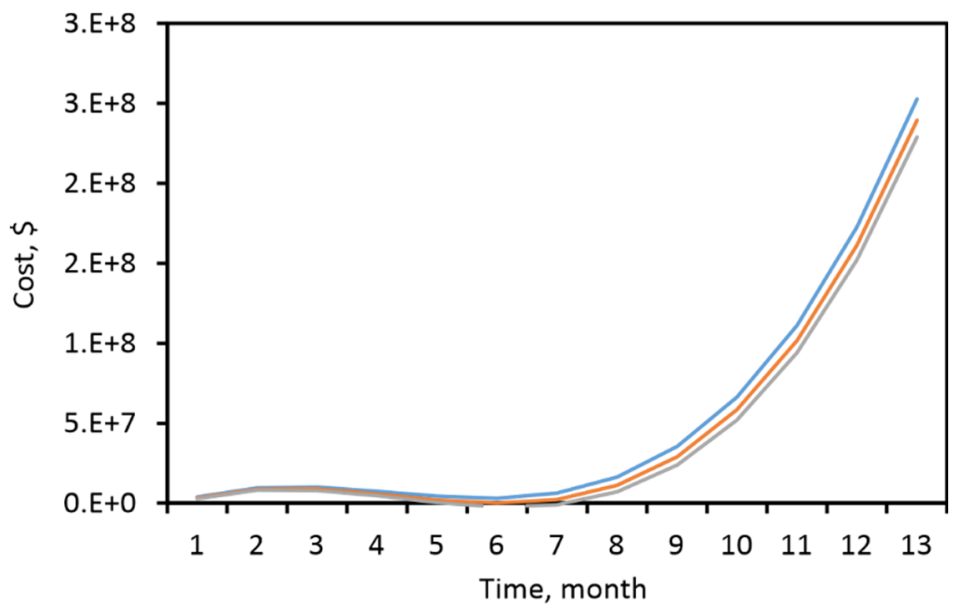

— Triangular fuzzy number —_ Parabolic fuzzy number

— Trapezoidal number

Fig. 2. Graphical representation of the results presented in Table 5

Table 6. Optimal values corresponding to various fuzzy lead times when the deterioration follows triangular distribution

\begin{tabular}{|c|c|c|c|c|c|}
\hline \multicolumn{2}{|c|}{ For triangular fuzzy number } & \multicolumn{2}{c|}{ For parabolic fuzzy number } & \multicolumn{2}{c|}{ For trapezoidal number } \\
\hline $\begin{array}{c}t_{1} \\
{[\text { months }]}\end{array}$ & $\begin{array}{c}t_{1} \\
{[\$]}\end{array}$ & $\begin{array}{c}T C \\
{[\text { months] }}\end{array}$ & $\begin{array}{c}t_{1} \\
{[\$]}\end{array}$ & $\begin{array}{c}T C \\
{[\text { months] }}\end{array}$ & {$[\$]$} \\
\hline 5 & 9946000 & 5 & 7168000 & 5 & 4973000 \\
\hline
\end{tabular}

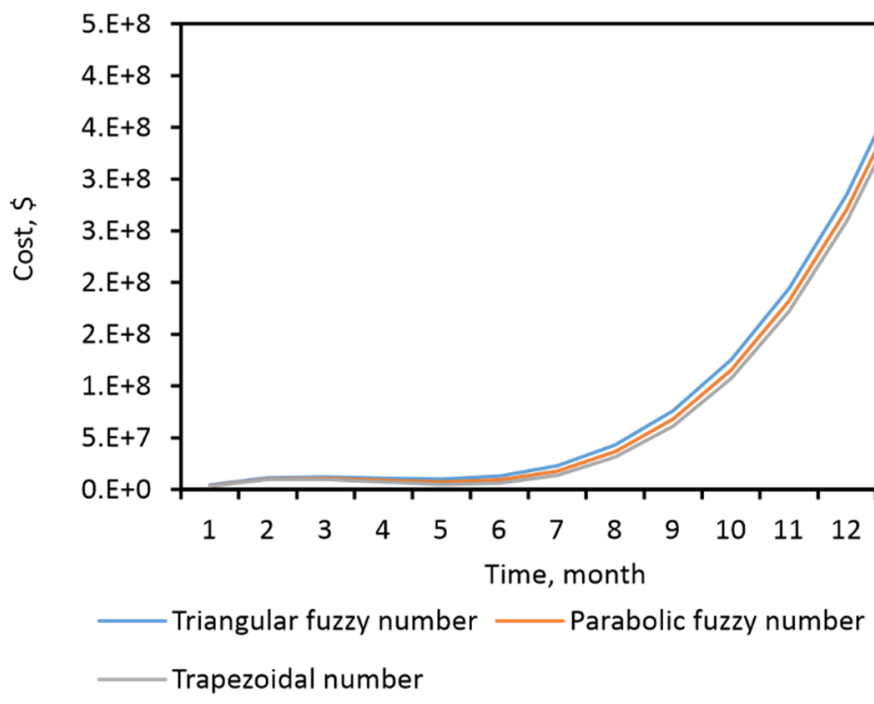

Fig. 3. Graphical representation of the results obtained in Table 6 
Table 7. Optimal values corresponding to different fuzzy lead times when the deterioration follows uniform distribution

\begin{tabular}{|c|c|c|c|c|c|}
\hline \multicolumn{2}{|c|}{ For triangular fuzzy number } & \multicolumn{2}{c|}{ For parabolic fuzzy number } & \multicolumn{2}{c|}{ For trapezoidal number } \\
\hline $\begin{array}{c}t_{1} \\
\text { [months }]\end{array}$ & $\begin{array}{c}T C \\
{[\$]}\end{array}$ & $\begin{array}{c}t_{1} \\
{[\text { months }]}\end{array}$ & $\begin{array}{c}T C \\
{[\$]}\end{array}$ & $\begin{array}{c}t_{1} \\
{[\text { months }]}\end{array}$ & $\begin{array}{c}T C \\
{[\$]}\end{array}$ \\
\hline 5 & 8937000 & 5 & 6251000 & 5 & 4128000 \\
\hline
\end{tabular}

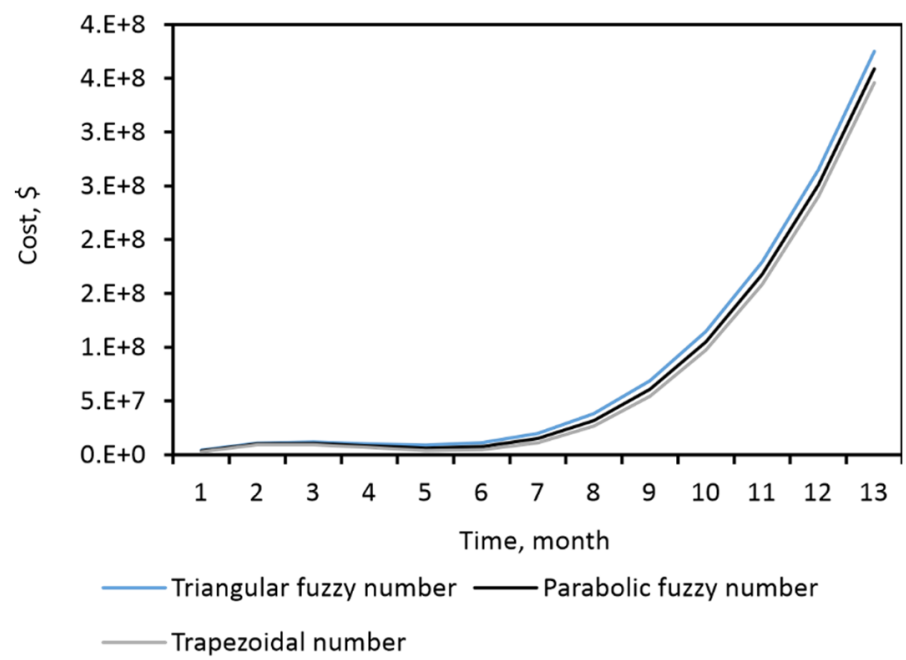

Fig. 4. Graphical representation of the results presented in Table 7

\section{Sensitivity analysis}

Table 8 . Sensitivity analysis of the proposed model; $\left(t_{1}, T C\right)$ values for various $\theta$ distributions and $L$ being triangular, parabolic, and trapezoidal fuzzy numbers

\begin{tabular}{|c|c|c|c|c|c|}
\hline \multicolumn{6}{|c|}{$\theta$ follows beta distribution } \\
\hline \multirow[b]{2}{*}{ Parameter } & \multirow{2}{*}{\multicolumn{2}{|c|}{ Change }} & \multicolumn{3}{|c|}{$L$} \\
\hline & & & \multirow{2}{*}{$\begin{array}{c}\begin{array}{c}\text { Triangular } \\
\text { fuzzy number }\end{array} \\
4\end{array}$} & \multirow{2}{*}{$\begin{array}{c}\begin{array}{c}\text { Parabolic } \\
\text { fuzzy number }\end{array} \\
5\end{array}$} & \multirow{2}{*}{$\begin{array}{l}\text { Trapezoidal } \\
\text { fuzzy number } \\
6\end{array}$} \\
\hline 1 & 2 & 3 & & & \\
\hline \multirow{5}{*}{$h$} & $50 \%$ & 3 & \multirow{5}{*}{$(6,3194000)$} & \multirow{5}{*}{$(6,129200)$} & \multirow{10}{*}{$T C$ negative } \\
\hline & $25 \%$ & 2.5 & & & \\
\hline & $0 \%$ & 2 & & & \\
\hline & $-25 \%$ & 1.5 & & & \\
\hline & $-50 \%$ & 1 & & & \\
\hline \multirow{5}{*}{$A$} & $50 \%$ & 150 & $(6,4749000)$ & $(6,152200)$ & \\
\hline & $25 \%$ & 125 & $(6,3971000)$ & $(6,140700)$ & \\
\hline & $0 \%$ & 100 & $(6,3194000)$ & $(6,129200)$ & \\
\hline & $-25 \%$ & 75 & $(6,2416000)$ & $(6,117600)$ & \\
\hline & $-50 \%$ & 50 & $(6,1638000)$ & $(6,106100)$ & \\
\hline
\end{tabular}


Table 8 continued

\begin{tabular}{|c|c|c|c|c|c|}
\hline 1 & 2 & 3 & 4 & 5 & 6 \\
\hline \multirow{5}{*}{$c_{s}$} & $50 \%$ & 7.5 & $(6,3193000)$ & \multirow{2}{*}{$(6,129100)$} & \multirow{15}{*}{$T C$ negative } \\
\hline & $25 \%$ & 6.25 & \multirow{4}{*}{$(6,3194000)$} & & \\
\hline & $0 \%$ & 5 & & \multirow{3}{*}{$(6,129200)$} & \\
\hline & $-25 \%$ & 3.75 & & & \\
\hline & $-50 \%$ & 2.5 & & & \\
\hline \multirow{5}{*}{ C } & $50 \%$ & 75 & $(6,3244000)$ & $(6,179100)$ & \\
\hline & $25 \%$ & 62.5 & $(6,3219000)$ & $(6,154100)$ & \\
\hline & $0 \%$ & 50 & $(6,3194000)$ & $(6,129200)$ & \\
\hline & $-25 \%$ & 37.5 & $(6,3169000)$ & $(6,104200)$ & \\
\hline & $-50 \%$ & 25 & $(6,3144000)$ & $(6,79200)$ & \\
\hline \multirow{5}{*}{$c_{d}$} & $50 \%$ & 1.125 & \multirow{3}{*}{$(6,3194000)$} & $(6,129500)$ & \\
\hline & $25 \%$ & $\begin{array}{c}0.937 \\
5 \\
\end{array}$ & & $(6,129300)$ & \\
\hline & $0 \%$ & 0.75 & & $(6,129200)$ & \\
\hline & $-25 \%$ & $\begin{array}{c}0.562 \\
5\end{array}$ & \multirow[t]{2}{*}{$(6,3193000)$} & $(6,129000)$ & \\
\hline & $-50 \%$ & 0.375 & & $(6,128800)$ & \\
\hline \multicolumn{6}{|c|}{$\theta$ follows triangular distribution } \\
\hline \multirow[b]{2}{*}{ Parameter } & \multirow{2}{*}{\multicolumn{2}{|c|}{ Change }} & & $L$ & \\
\hline & & & $\begin{array}{c}\text { Triangular } \\
\text { fuzzy number }\end{array}$ & $\begin{array}{c}\text { Parabolic } \\
\text { fuzzy number }\end{array}$ & $\begin{array}{c}\text { Trapezoidal } \\
\text { fuzzy number }\end{array}$ \\
\hline \multirow{5}{*}{$h$} & $50 \%$ & 3 & \multirow{5}{*}{$(5,9946000)$} & \multirow{5}{*}{$(5,7168000)$} & \multirow{5}{*}{$(5,4973000)$} \\
\hline & $25 \%$ & 2.5 & & & \\
\hline & $0 \%$ & 2 & & & \\
\hline & $-25 \%$ & 1.5 & & & \\
\hline & $-50 \%$ & 1 & & & \\
\hline \multirow{5}{*}{$A$} & $50 \%$ & 150 & $(5,14870000)$ & $(5,10710000)$ & $(5,4173000)$ \\
\hline & $25 \%$ & 125 & $(5,12410000)$ & $(5,8937000)$ & $(5,6193000)$ \\
\hline & $0 \%$ & 100 & $(5,9946000)$ & $(5,7168000)$ & $(5,4973000)$ \\
\hline & $-25 \%$ & 75 & $(5,7482000)$ & $(5,5399000)$ & $(5,3750000)$ \\
\hline & $-50 \%$ & 50 & $(5,5019000)$ & $(5,3631000)$ & $(5,2533000)$ \\
\hline \multirow{5}{*}{$c_{S}$} & $50 \%$ & 7.5 & $(5,9945000)$ & \multirow{15}{*}{$(5,7168000)$} & \multirow{15}{*}{$(5,4973000)$} \\
\hline & $25 \%$ & 6.25 & \multirow{12}{*}{$(5,9946000)$} & & \\
\hline & $0 \%$ & 5 & & & \\
\hline & $-25 \%$ & 3.75 & & & \\
\hline & $-50 \%$ & 2.5 & & & \\
\hline \multirow{5}{*}{ C } & $50 \%$ & 75 & & & \\
\hline & $25 \%$ & 62.5 & & & \\
\hline & $0 \%$ & 50 & & & \\
\hline & $-25 \%$ & 37.5 & & & \\
\hline & $-50 \%$ & 25 & & & \\
\hline \multirow{5}{*}{$c_{d}$} & $50 \%$ & 1.125 & & & \\
\hline & $25 \%$ & 0.9375 & & & \\
\hline & $0 \%$ & 0.75 & & & \\
\hline & $-25 \%$ & 0.5625 & \multirow{2}{*}{$(5,9945000)$} & & \\
\hline & $-50 \%$ & 0.375 & & & \\
\hline
\end{tabular}


Table 8 continued

\begin{tabular}{|c|c|c|c|c|c|}
\hline \multicolumn{6}{|c|}{$\theta$ follows uniform distribution } \\
\hline 1 & 2 & 3 & 4 & 5 & 6 \\
\hline \multirow{5}{*}{$h$} & $50 \%$ & 3 & \multirow{5}{*}{$(5,8937000)$} & \multirow{5}{*}{$(5,6251000)$} & \multirow{5}{*}{$(5,4128000)$} \\
\hline & $25 \%$ & 2.5 & & & \\
\hline & $0 \%$ & 2 & & & \\
\hline & $-25 \%$ & 1.5 & & & \\
\hline & $-50 \%$ & 1 & & & \\
\hline \multirow{5}{*}{$A$} & $50 \%$ & 150 & $(5,13360000)$ & $(5,9331000)$ & $(5,6146000)$ \\
\hline & $25 \%$ & 125 & $(5,11150000)$ & $(5,7791000)$ & $(5,5137000)$ \\
\hline & $0 \%$ & 100 & $(5,8937000)$ & $(5,6251000)$ & $(5,4128000)$ \\
\hline & $-25 \%$ & 75 & $(5,6726000)$ & $(5,4712000)$ & $(5,3119000)$ \\
\hline & $-50 \%$ & 50 & $(5,4515000)$ & \multirow{6}{*}{$(5,3172000)$} & $(5,2111000)$ \\
\hline \multirow{5}{*}{$c_{s}$} & $50 \%$ & 7.5 & \multirow{6}{*}{$(5,8937000)$} & & \multirow{5}{*}{$(5,4128000)$} \\
\hline & $25 \%$ & 6.25 & & & \\
\hline & $0 \%$ & 5 & & & \\
\hline & $-25 \%$ & 3.75 & & & \\
\hline & $-50 \%$ & 2.5 & & & \\
\hline \multirow{5}{*}{$C$} & $50 \%$ & 75 & & $(5,6301000)$ & $(5,4178000)$ \\
\hline & $25 \%$ & 62.5 & $(5,8962000)$ & $(5,6276000)$ & $(5,4153000)$ \\
\hline & $0 \%$ & 50 & $(5,8937000)$ & $(5,6251000)$ & $(5,4128000)$ \\
\hline & $-25 \%$ & 37.5 & $(5,8912000)$ & $(5,6226000)$ & $(5,4103000)$ \\
\hline & $-50 \%$ & 25 & $(5,8887000)$ & $(5,6201000)$ & $(5,4078000)$ \\
\hline \multirow{5}{*}{$c_{d}$} & $50 \%$ & 1.125 & $(5,8938000)$ & $(5,6252000)$ & $(5,4129000)$ \\
\hline & $25 \%$ & 0.9375 & \multirow{4}{*}{$(5,8937000)$} & $(5,6525000)$ & \multirow{4}{*}{$(5,4128000)$} \\
\hline & $0 \%$ & 0.75 & & & \\
\hline & $-25 \%$ & 0.5625 & & $(5,6251000)$ & \\
\hline & $-50 \%$ & 0.375 & & & \\
\hline
\end{tabular}

Changes in the values of system parameters may take place due to uncertainties in any decision-making situation. So, to study the implications of these changes, it is essential to perform sensitivity analysis. Here we study the effects of changes in the values of system parameters on the optimal values of time of replenishment and total cost when the deterioration rate follows different probability distributions. As based on our numerical calculation presented in Table 8, we have the following observations.

- There is no effect on the time of replenishment when lead time is taken as triangular, parabolic, and trapezoidal numbers under the assumptions of different probability distributions for the deterioration rate.

- The optimal total cost (TC) is not acceptable due to changes in system parameters when lead time is taken as the trapezoidal fuzzy number and $T C$ remains unchanged under beta distribution for deterioration rate as $h$ is increased or decreased when lead time is taken triangular, parabolic fuzzy number. $50 \%$ changes in the value of $h, T C$ changes with lead time as triangular, parabolic, and trapezoidal fuzzy number in the case 
of triangular distribution for deterioration rate. For any change in the value of $h, T C$ remains unaltered with lead time as triangular, parabolic, and trapezoidal fuzzy number, in the case of uniform distribution for the deterioration rate.

- It is clear from the sensitivity analysis that there is a direct relation of optimal $T C$ with the parameters $A, C$. That is, the maximum value of $A$ and $C$ will maximize $T C$. To avoid such type of situation, the decision manager should purchase the goods at a reasonable price to minimize $T C$.

- $50 \%$ decrease in unit deterioration cost minimizes $T C$ when lead time is a parabolic fuzzy number under beta distribution, whereas the same percentage of decrease in unit deterioration cost does not minimize $T C$ when lead time is triangular, parabolic, and trapezoidal fuzzy number under triangular and uniform distributions.

- Our next observation is on optimal $T C$ due to changes in the value of unit shortage $\operatorname{cost} c_{s} . T C$ is minimal due to $50 \%$ positive changes on the said parameter when lead time is taken as a triangular fuzzy number under beta and triangular distributions.

\section{Conclusion}

For the first time, the inventory model for deteriorating items with negative exponential demand is developed in the presence of probabilistic deterioration and fuzzy lead time. In this study, the model is formulated and solved with non-zero lead time and different probabilistic deterioration to obtain optimal values. Following this, the model is considered in a fuzzy environment. Corresponding to different probabilistic distributions, lead time was considered as different fuzzy numbers under three different cases of probabilistic deterioration, and optimal values may be compared. The developed model might be helpful for retailers in dealing with the problems of items like fashionable goods, a certain brand of medicine, etc., due to their demand pattern. The present investigation may be further extended with new assumptions like salvage cost, preservation technology, and variable holding cost.

\section{Acknowledgement}

The authors express their gratitude to anonymous referees for their constructive comments and indications for improvements.

\section{References}

[1] BHUNIA A.K., SHAIKH A., A deterministic inventory model for deteriorating items with selling price dependent demand and three-parameter Weibull distributed deterioration. Int. J. Ind. Eng. Comp., 2104, 5 (3), 497-510. 
[2] CHANDRA S., An inventory model with ramp type demand, time varying holding cost and price discount on backorders, Unc. Supp. Chain Manage., 2017, 5 (1), 51-58.

[3] Chou S.Y., Julian P.C., Hung K.C., A note on fuzzy inventory model with storage space and budget constraints, Appl. Math. Model., 2009, 33 (11), 4069-4077.

[4] DE S.K., SANA S.S., Fuzzy order quantity inventory model with fuzzy shortage quantity and fuzzy promotional index, Econ Model., 2013, 31, 351-358.

[5] DAS D., RoY A., KAR S., Improving production policy for deteriorating items under partial delay in payment with stock dependent demand, Comp. Math. Appl., 201060 (7),1973-1985.

[6] HaLIM M.A., Weibull distributed deteriorating inventory model with ramp type demand and fully backlogged shortages, Asian J. Math. Comp. Sci. Res., 2017, 19 (3), 148-157.

[7] JAGGi C.K., PAREEK S., KhANNA A., NiDHI N., Optimal replenishment policy for fuzzy inventory model with deteriorating items and allowable shortages under inflationary conditions, Yugoslavian J. Oper. Res., 2016, 26 (4), 507-526.

[8] Khurana D., TAYAl S., Singh R.S., An EPQ model for deteriorating items with variable demand rate and allowable shortages, Int. J. Oper. Res. Math., 2018, 12 (1), 117-128.

[9] KAZEMI N., EHSANI E., JABER M.Y., An inventory model with back orders with fuzzy parameters and decision variable, Int. J. Appr. Res., 2010, 51 (8), 964-972.

[10] Lotfi R., Weber G.W., SAJAdifar S.M., MARDAni N., Interdependent demand in the two-period newsvendor problem, J. Ind. Manage. Opt., 2018, 777-792.

[11] Mishra V.K., Singh L.S., Kumar R., An inventory model for deteriorating items with time-dependent demand and time-varying holding cost under partial backlogging, J. Ind. Eng. Int., 2013, 9 (1), 4.

[12] Misra S., Misra U., Misra G., An inventory model for inflation induced demand and Weibull deteriorating items, Int. J. Adv. Eng. Technol., 2012, 4 (1), 176-182.

[13] MaITI M.K., Fuzzy inventory model with two warehouses under possibility measure on fuzzy goal, Eur. J. Oper. Res., 2008, 188 (3), 746-774.

[14] Mandal N.K., Roy T.K., A displayed inventory model with L-R fuzzy number, Fuzzy Opt. Dec. Mak., 2006, 5 (3), 227-243.

[15] Mandal M.K., Roy T.K., Maiti M., Multi-objective inventory model with three constraints: a geometric programming approach, Fuzzy Set Syst., 2005, 150 (1), 87-106.

[16] Panda S., Saha S., ModaK M.N., Sana S.S., A volume flexible deteriorating inventory model with price sensitive demand, Tékh., Rev. Appl. Manage. Stud., 2017, 15 (2), 117-123.

[17] Palanivel M., Priyan S., Uthayakumar R., An inventory model with finite replenishment, probabilistic deterioration and permissible delay in payments, J. Manage. Anal., 2015, 2 (3), 254-279.

[18] Reddy M.S., VenKateswarlu R., Perishable inventory models for stock dependent quadratic demand under inflation, IOSR J. Math., 2016, 12 (2), 64-70.

[19] Roy A., Fuzzy inventory model for deteriorating items with price dependent demand, Int. J. Manage. Sci. Eng. Manage., 2015, 10 (4), 237-241.

[20] Roy A., MAITI M.K., KAR S., MAITI M., An inventory model for deteriorating items with displayed stock dependent demand under fuzzy inflation and time discounting over a random planning horizon, Appl. Math. Model., 2009, 3 (2), 744-759.

[21] RoY A., KAR S., MAITI M., A deteriorating multi-item inventory model with fuzzy costs and resources based on two different defuzzification techniques, Appl. Math. Model., 2008, 32 (2), 208-223.

[22] SEN N., SaHa S., An inventory model for deteriorating items with time dependent holding cost and shortages under permissible delay in payment, Int. J. Proc. Manage., 2018, 11, (4), 518-531.

[23] Sнан N.H., NAIK M.K., Inventory policies for deteriorating items with time price backlog dependent demand, Int. J. Syst. Sci. Oper. Log., 2020, 7 (1), 76-89.

[24] SHAH N.H., NAIK M., EOQ model for deteriorating item under full advance payment availing of discount when demand is price sensitive, Int. J. Sup. Chain Oper. Res., 2018, 3 (2), 163-197. 
[25] SAHA S., SEN N., A study on inventory model with negative exponential demand and probabilistic deterioration under backlogging, Unc. Sup. Chain Manage., 2017, 5 (2), 77-88.

[26] Saha S., Chakrabarti T., Fuzzy inventory model for deteriorating items in a supply chain system with price dependent demand and without backorder, Am. J. Eng. Res., 2017, 6 (6), 183-187.

[27] Sen N., Nath B.K., SAHa S., A fuzzy inventory model for deteriorating items based on different defuzzification techniques, Am. J. Math. Stat., 2016, 6 (3), 128-137.

[28] SHARMila D., UthaYAKUMAR R., Inventory model for deteriorating items involving fuzzy with shortages and exponential demand, Int. J. Sup. Oper. Manage., 2015, 2 (3), 888-904.

[29] SARKAR B., SARKAR S., Variable deterioration and demand an inventory model, Econ. Mod., 2013, $31,548-556$.

[30] TRIPATHI R.P., Inventory model with time dependent demand rate under inflation when supplier credit linked to ordered quantity, Int. J. Bus. Inf. Tech., 2011, 3, 174-183.

[31] TRIPATHI R.P., PAREEK S., KAUR M., Inventory model with power demand and inventory-induced demand with holding cost function, Am. J. Appl. Sci., 2017, 14 (6), 607-613. 